\title{
Solid-Phase Extraction and Determination of Trace Aroma and Flavour Components in Cider by GC-MS
}

\author{
J. J. Mangas ${ }^{1}$ / M.P. González 1 R. Rodríguez ${ }^{1}$ / D. Blanco*2 \\ ${ }^{1}$ Instituto de Experimentación y Promoción Agraria, 33300 Villaviciosa, Spain \\ ${ }^{2}$ Departamento de Química Física y Analítica, Universidad de Oviedo, 33006 Oviedo, Spain
}

\section{Key Words}

Gas chromatography-mass spectrometry

Solid-phase extraction

Cider

Aroma and flavour

\section{Summary}

Minor volatile compounds are responsible for the aromas of cider. A simple technique for the analysis of these components is described based on solid-liquid phase extraction followed by quantitation by gas chromatography-mass spectrometry(GC-MS). The method is quantitative for analysis of alcohols, esters, lactones, phenols, and medium and long chain-length fatty acid.

\section{Introdaction}

Volatile components play an important role in the quality of cider, therefore it is very important to understand the formation of chemical flavours during its making. The analysis of flavour constituents, particularly the lipophilic compounds, requires their removal from the media, their purification and concentration. Different methods have been used in several complex media in order to achieve this, namely: the headspace technique [1], steam distillation and supercritical fluid extraction [2], trapping over porous polymer [3-4], solid-liquid extraction over resins [5-7], purge-extraction techniques [8], simultaneous distillation-extraction [9] and batch and continuous solvent extraction [10].

Although the static headspace technique is a very attractive method in the analysis of food and beverage aromas and volatile pollutants, its primary disadvantage is that of low sensitivity with respect to low volatile components and trace compounds. On the other hand, sensitivity may be increased by purge and trap techniques. Simultaneous distillation-extraction is not time-consuming, but artefact formation due to thermally induced changes is possible. Likewise, distillation and liquid-li- quid extraction are well fitted for monitoring the components responsible for aroma, but are time-consuming and also, the stability of the emulsion produced is quite significant, making separation of the phases difficult. The absorption of analytes onto hydrophobic resins, such as, XAD-2 and $\mathrm{C}_{18}$, has been widely employed [11] because of their power to extract organic components from aqueous media, but a high level of interference by ethanol (the major component in alcoholic beverages) occurs. An alternative system is to employ hydrophilic resins to retain water and high polarity compounds and to then elute the low polarity organic components with appropriate solvents.

The aim of this work was to evaluate a solid-phase extraction procedure in order to develop a quantitative method for monitoring flavour components of cider.

\section{Experimental}

Analytical-grade samples of different standards and solvents were from Fluka-Chemika (Buchs, Switzerland), Aldrich-Chemie (Steinheim, Germany), Sigma (St. Louis, USA), and Lab-Scan (Barcelona, Spain) and were used without further purification.

\section{Raw Material}

Two typical ciders, supplied by traditional makers from the Region of Asturias, with different sensory profiles (fruity and sharp aromas) were employed in this study. The cider was prepared from the juice of a mixture of cider apples with different sensory properties endowing the resulting juice with an overall acidic nature. The processing of this mixture included the following steps: milling with a hammer mill, slow pressing and fermentation by wild yeast.

\section{Extraction}

Extrelut-20 columns (Merck, Darmstadt, Germany) filled with large-pore kieselguhr of granular structure supported by a frit glass for the extraction of lipophilic compounds of cider, were employed. The sample $(20 \mathrm{~mL})$ and the internal standard $\left(16.69 \mathrm{mg} \mathrm{L}^{-1} 2\right.$ - 
ethyl-1-hexanol), adjusted to $\mathrm{pH} 3.00$ with hydrochloric acid, were degassed using an ultrasonic bath for $3 \mathrm{~min}$ and percolated through the kieselguhr and allowed to soak for $15 \mathrm{~min}$ until totally absorbed. Immediately after, $80 \mathrm{~mL}$ dichloromethane were eluted through the column by manifold vacuum for $30 \mathrm{~min}$. Anhydrous sodium sulphate was used to dehydrate the organic phase. Next, $50 \mathrm{~mL}$ organic extract were collected and then concentrated. A helical gas flow (nitrogen) by vortexing action (Turbo Vap $^{T M}$ Evaporator-Zymark) was employed for solvent removal to a final volume of $0.5 \mathrm{~mL}$; which was monitored using microswitch and optical sensing. The concentration temperature was kept at $30^{\circ} \mathrm{C}$ by a water bath.

\section{GC-MS}

GC-MS was on a Hewlett-Packard 5970A mass selective detector (MSD) interfaced with a Hewlett-Packard 5790 gas chromatograph (Palo Alto, CA) fitted with a column (FFAP, $50 \mathrm{~m} \times 0.22 \mathrm{~mm}$ i.d.; phase thickness, $0.33 \mu \mathrm{m}$ ) inserted into a split/splitless injector (splitless time: $1 \mathrm{~min}$ ) and terminated at the MSD. Chromatographic conditions were as follows: initial temperature, $40^{\circ} \mathrm{C}$; program rate, $3{ }^{\circ} \mathrm{C} \mathrm{min}^{-1}$; final temperature, $220^{\circ} \mathrm{C}$; injector and detector temperature, $250^{\circ} \mathrm{C}$; MS interface temperature, $160^{\circ} \mathrm{C}$; carrier gas, He at $1 \mathrm{~mL}$ min $^{-1}$. Injection $(1 \mu \mathrm{L})$ was by means of an autosampler. Analyses were performed in the electron impact (EI) mode and ionisation voltage was $70 \mathrm{eV}$. Both scan and ion-monitoring modes for detection were chosen.

\section{Results and Discussion}

The GC-MS method used enabled us to identify 36 cider aroma components in Asturian ciders with a confidence level of more than $72 \%$ : including twelve alcohols (1propanol, isobutanol, 2-propenol, 1-butanol, amyl alcohols, 3-ethoxy-1-propanol, 1-hexanol, 2,3-butanediol, 2-(methylthio)-1-propanol, benzyl alcohol, 2-phenylethanol, tyrosol); eight esters (ethyl lactate, diethyl succinate, ethyl caproate, ethyl caprylate, ethyl caprate, ethyl laurate, ethyl myristate, ethyl palmitate); two ketones (acetoin, $\gamma$-butyrolactone); two phenols (4ethylguaiacol, 4-ethylphenol) and twelve organic acids (acetic, propanoic, isobutyric, butanoic, caproic, caprylic, capric, benzoic, lauric, phenylacetic, myristic, palmitic).

Quantitation of the aroma compounds was by using the internal standard method. 2-Ethyl-1-hexanol was chosen as the standard for all volatile compounds, and its concentration in the initial solutions was $16.69 \mathrm{mg} \mathrm{L}^{-1}$. The use of undecanoic acid as standard for volatile compounds eluted in the last part of the chromatogram did not improve the results obtained. The relative retention time, the response (peak area) ratio, and relative response factor of each volatile against 2-ethyl-1hexanol were determined at concentrations of each component ranging from the quantitation limits to threetimes the amount present in cider. The relative response factor was obtained by dividing the peak area of each volatile by the peak area of the internal standard. The relative response factor divided by the concentration ratio (volatile compound:2-ethyl-1-hexanol) yielded the relative response factors for each compound. These response factors were around 1.4-1.5 for ethyl esters of long-chain fatty acids and diethylsuccinate, about 1.1 for acetate esters and aromatic alcohols, close to 1 for ethyl esters of the middle aroma fraction and long-chain monocarboxylic acids, close to 0.8 for higher alcohols and near 0.5 for $\gamma$-butyrolactone, 2-3 butanediol and ethyl lactate.

Recovery studies were carried out to determine the accuracy of the method. Known amounts (between $50 \%$ and $150 \%$ of the amounts found in cider) of each volatile belonging to the different chemical types above, were added to a variety of samples and the resulting spiked samples were subjected to the entire analytical sequence. All analyses were carried out in triplicate at three concentration levels. For the recovery studies, the mass spectrometer was operated in the selected-ion monitoring mode. The characteristic ions, namely: $\mathrm{m} / \mathrm{z}=60\left(\mathrm{C}_{2} \mathrm{H}_{4} \mathrm{O}_{2}{ }^{+}\right)$and $73\left(\mathrm{C}_{3} \mathrm{H}_{5} \mathrm{O}_{2}{ }^{+}\right)$for aliphatic organic acids, $\mathrm{m} / \mathrm{z}=88\left(\mathrm{C}_{4} \mathrm{H}_{8} \mathrm{O}_{2}{ }^{+}\right)$for fatty acid ethyl esters, $\mathrm{m} / \mathrm{z}=43\left(\mathrm{C}_{2} \mathrm{H}_{3} \mathrm{O}^{+}\right)$for acetates, $[\mathrm{M}-15]^{+}$for 4ethyl phenol derivatives, $[\mathrm{M}-31]^{+}$for $\beta$-hydroxyethyl phenol derivatives and $[M-44]^{+}$for lactones, were employed in quantitative studies. The alcohols and ketones were quantified by means of appropriate ions in order to minimise interference of other analytes.

The average recoveries (Table I) for alcohols, hydroxyacid esters, lactones, phenols and fatty acids are acceptable with a relative standard deviation of less than

Table I. Recovery of some volatile compounds added to various ciders.

\begin{tabular}{|lr|}
\hline Compound & \% Recovery \pm RSD \\
\hline 1-Propanol & $104.83 \pm 2.95$ \\
Isobutanol & $78.73 \pm 2.45$ \\
Amyl alcohols & $100.03 \pm 1.40$ \\
1-Butanol & $102.01 \pm 5.30$ \\
2,3(R,R-S,S)-Butanediol & $12.30 \pm 4.73$ \\
2,3-Butanediol(meso form) & $5.07 \pm 3.26$ \\
2-Phenylethanol & $105.33 \pm 5.39$ \\
Acetoin & $84.2 \pm 6.20$ \\
Ethyl lactate & $88.31 \pm 0.98$ \\
Diethyl succinate & $90.32 \pm 4.20$ \\
Benzyl alcohol & $92.10 \pm 2.50$ \\
y-Butyrolactone & $103.00 \pm 3.96$ \\
3-(Methylthio)-1-propanol & $77.50 \pm 6.53$ \\
Ethyl caproate & $65.70 \pm 4.80$ \\
Ethyl caprylate & $69.23 \pm 4.40$ \\
Ethyl caprate & $68.53 \pm 4.56$ \\
Ethyl laurate & $67.54 \pm 7.58$ \\
Propanoic acid & 0 \\
2-Phenylethyl acetate & $92.00 \pm 3.15$ \\
Caprylic acid & $94.82 \pm 6.01$ \\
4-Ethylphenol & $87.51 \pm 3.42$ \\
Isoamyl acetate & $83.00 \pm 5.00$ \\
Capric acid & $94.37 \pm 4.06$ \\
Benzoic acid & $110.41 \pm 4.19$ \\
\hline
\end{tabular}




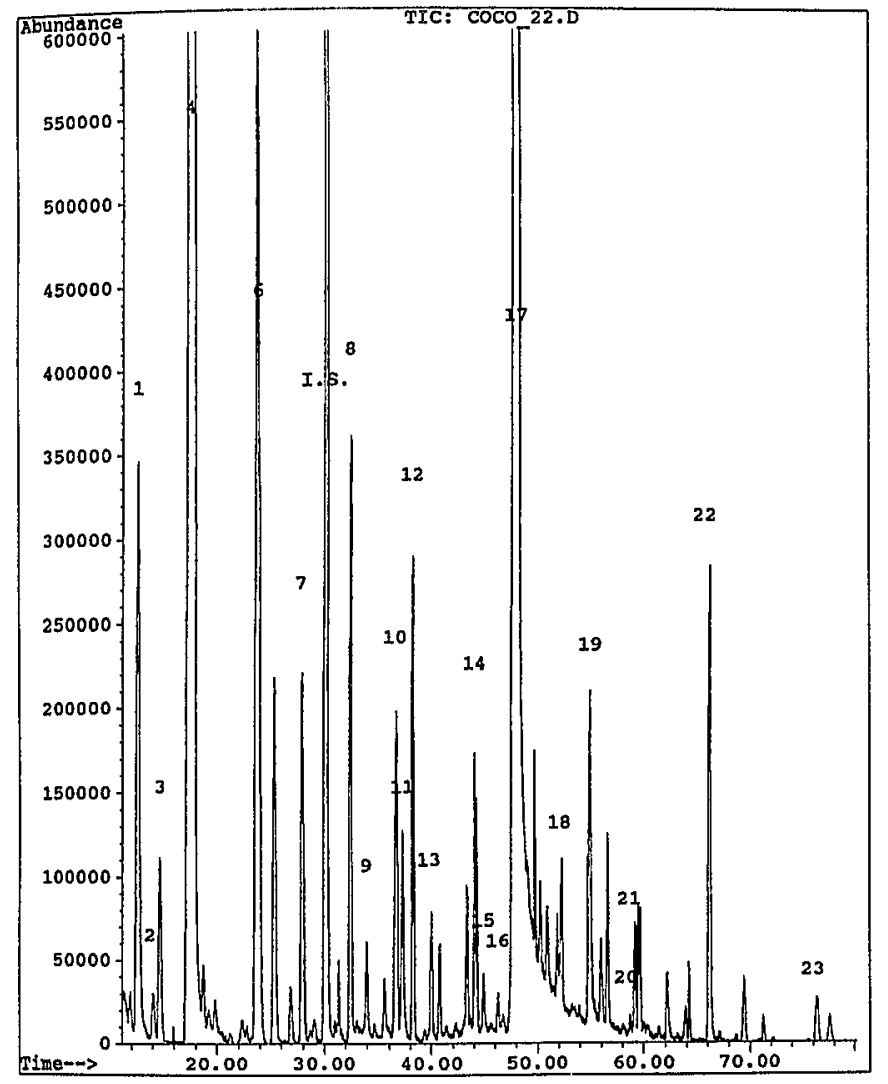

Figure 1

Total ion chromatogram of volatile compounds in fruity cider; $\mathbf{1}=$ isobutanol; $\mathbf{2}=$ isoamyl acetate; $\mathbf{3}=$ butanol; 4 = amyl alcohols; 6 = ethyl lactate; 7 = ethyl caprylate; $8=2,3$ (R,R and/or S,S)-butanediol; $9=2,3$-butanediol (meso form); $\mathbf{1 0}=$ ethyl caprate; $\mathbf{1 1}=$ dihydro2(3H)-furanone; 12 = diethyl succinate; $13=3$-(methylthio)-1-propanol; 14 = 2-phenylethyl acetate; 15 = ethyl laurate; 16 = benzyl alcohol; 17 = 2-phenylethanol; 18 = caprylic acid; 19 = 4-ethylphenol; $\mathbf{2 0}$ = ethyl palmitate; $\mathbf{2 1}$ = capric acid; $\mathbf{2 2}$ = lauric acid; $\mathbf{2 3}$ = myristic acid.

$6 \%$, indicating that the proposed method is suitable for their analysis. However, recoveries for fatty acid ethyl esters and butanediol are lower, and organic acids of low molecular mass, for example, propanoic acid, were not extracted. The short-chain esters were not detected under our experimental conditions because they are associated with the solvent front.

This chromatographic method was used for studying the flavour of two ciders with different sensory profiles: fruity and sharp aromas (Figures 1 and 2). As regards to higher alcohol levels, more amyl alcohols (typical nosecatching aromas) [12], 1-butanol, and isobutanol were detected in the cider with a sharp aroma (Table II); however, the concentration of 2-phenylethanol, an alcohol associated with a scented aroma according to Williams' criteria [12], was higher in the fruity cider. On the other hand, although benzyl alcohol or phenylmethanol is not a common by-product in fermentation by yeast, it was found in both ciders monitored. The concentration of this alcohol was higher in the cider with sharp aromas. Isoamyl acetate, long associated

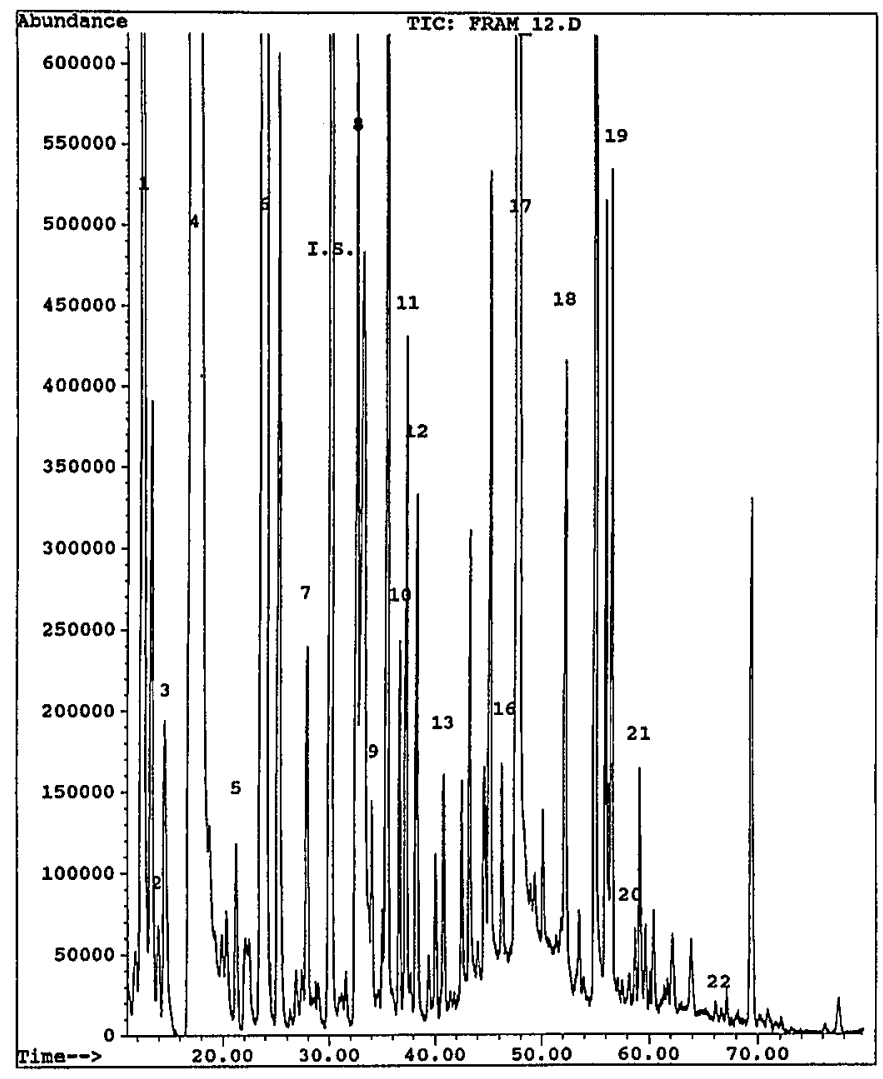

Figure 2

Total ion chromatogram of volatile compounds in sharp cider; $\mathbf{1}=$ isobutanol; 2 = isoamyl acetate; $3=1$-butanol; 4 = amyl alcohols; 5 = acetoin; 6 = ethyl lactate; $7=$ ethyl caprylate; $8=2,3(\mathrm{R}, \mathrm{R}$ and/or $\mathrm{S}, \mathrm{S}$ )-butanediol; $\mathbf{9}=2,3$-butanediol (meso form); $\mathbf{1 0}=$ ethyl caprate; $\mathbf{1 1}=$ dihydro-2(3H)-furanone; 12 = diethyl succinate; 13 = 3-(methylthio)-1-propanol; 16 = benzyl alcohol; $17=2$-phenylethanol; $18=$ caprylic acid; $\mathbf{1 9}=$ 4-ethylphenol; $\mathbf{2 0}=$ ethyl palmitate; $\mathbf{2 1}=$ capric acid; $\mathbf{2 2}$ = lauric acid.

with banana odour by chemists [13], is present in the ciders tested, but 2-phenylethyl acetate (an analyte associated with scented aromas) was only noticed in the fruity cider (Table II), which is due to a higher level of phenethyl alcohol. In relation to the ethyl esters of hydroxy and dicarboxylic acids, more ethyl lactate was detected in the sharp aroma cider, which may be due to esterification by lactic acid bacteria strains; the same diethyl succinate level was detected in both ciders. On the other hand, it has been pointed out that the contribution of ethyl lactate in alcoholic beverage aromas may be low because of their odour threshold values of $250 \mathrm{ppm}$ [14].

Several esters of long-chain fatty acids (ethyl esters of caprylic, capric, lauric and palmitic acids) were detected in the ciders; these have long been associated with fruity (the first members of this group), soap-scented (intermediate members of this group) and stearine ( $\mathrm{C}_{16}$ and $\mathrm{C}_{18}$ ester acids) aromas [15]; we found a higher level of ethyl esters of caprylic, capric, and lauric acids in the fruity cider than in the cider with sharp aroma (Table 
Table I. Content ( $\mathrm{mg} \mathrm{L}^{-1} \pm \mathrm{RSD}$ ) of volatile components of two ciders determined by solvent extraction and Extrelut resin.

\begin{tabular}{|c|c|c|c|}
\hline \multirow{2}{*}{ Component } & \multirow{2}{*}{$\underset{(\mathrm{min})}{\mathrm{R}_{\mathrm{t}}}$} & \multicolumn{2}{|c|}{ Cider } \\
\hline & & Fruity & Sharp \\
\hline 1-Butanol (3) & 14.65 & $2.24 \pm 4.09$ & $3.88 \pm 4.60$ \\
\hline Amyl alcohols (4) & 17.43 & $134.29 \pm 3.46$ & $170.85 \pm 5.50$ \\
\hline Isobutanol (1) & 12.59 & $7.73 \pm 3.37$ & $21.92 \pm 7.55$ \\
\hline 2-Phenylethanol (17) & 47.95 & $185.24 \pm 4.24$ & $57.30 \pm 5.90$ \\
\hline Benzyl alcohol (16) & 46.25 & $0.53 \pm 5.60$ & $1.54 \pm 8.83$ \\
\hline Ethyl lactate (6) & 23.68 & $11.86 \pm 4.80$ & $100.27 \pm 3.87$ \\
\hline Diethyl succinate(12) & 38.42 & $2.27 \pm 4.80$ & $2.32 \pm 3.28$ \\
\hline Ethyl caprylate (7) & 27.88 & $2.22 \pm 3.50$ & $1.92 \pm 6.43$ \\
\hline Ethyl caprate (10) & 36.73 & $3.39 \pm 7.80$ & $2.15 \pm 5.89$ \\
\hline Ethyl laurate (15) & 44.92 & $0.68 \pm 7.00$ & N.D. \\
\hline Ethyl palmitate(20) & 58.74 & $0.38 \pm 3.66$ & $0.66 \pm 4.14$ \\
\hline i-Amyl acetate (2) & 13.98 & $0.28 \pm 6.30$ & $0.58 \pm 9.50$ \\
\hline 2-Phenylethyl acetate (14) & 44.01 & $1.21 \pm 5.15$ & N.D. \\
\hline Dihydro-2(3H)-furanone (11) & 37.21 & $3.50 \pm 5.45$ & $7.90 \pm 2.94$ \\
\hline Acetoin (5) & 21.20 & N.D. & $1.92 \pm 4.88$ \\
\hline 2,3(R,R-S,S)-Butanediol (8) & 32.44 & $37.63 \pm 6.14$ & $90.89 \pm 4.84$ \\
\hline 2,3-Butanediol(meso form)(9) & 33.98 & $22.10 \pm 6.30$ & $75.34 \pm 7.92$ \\
\hline 3-(Methylthio)-1-propanol(13) & 40.00 & $1.41 \pm 8.88$ & $1.80 \pm 6.17$ \\
\hline 4-Ethylphenol (19) & 56.49 & $1.37 \pm 8.92$ & $3.33 \pm 5.56$ \\
\hline Caprylic acid (18) & 52.23 & $1.22 \pm 8.03$ & $3.51 \pm 4.39$ \\
\hline Capric acid (21) & 59.13 & $1.25 \pm 8.10$ & $2.20 \pm 2.42$ \\
\hline Lauric acid (22) & 66.16 & $4.88 \pm 5.00$ & $0.77 \pm 8.18$ \\
\hline Myristic acid (23) & 76.40 & $1.91 \pm 4.80$ & N.D. \\
\hline
\end{tabular}

N.D. $=$ not detected

II). However, a lower ethyl palmitate level was detected in the cider with a fruity aroma.

Certain typical compounds produced in the spoilage of fermented products were also monitored, namely acetoin, and 2,3 (S,S) and/or 2,3 (R,R) butanediol and butanediol, meso form. As seen in Table II, acetoin was only detected in the sharp aroma cider as well as a higher level of butanediol, which indicates a higher level of spoilage.

Acetaldehyde, long associated with sharp flavours [12], was quantified by the GC method optimised by the authors [16]; the above GC-MS technique could not be employed in acetaldehyde analysis because the solvent front did not allow accurate quantitation of this analyte. Between 20 and $45 \mathrm{ppm}$ acetaldehyde were detected in both ciders. The level of this component cannot, therefore, explain sensorial differences determined, since its sensory threshold is rather high, and hence small changes in acetaldehyde level can hardly exert an influence on the odour.

The concentration of $\gamma$-butyrolactone(dihydro-2(3H)furanone) was higher ( 2.25 times) in the sharp cider than in the fruity one. On the other hand, a higher concentration of 3-(methylthio)-1-propanol(methionol) was detected in the sharp cider. Methionol may be considered quantitatively as an important compound in alcoholic beverages; it has a typically sulphur flavour (cabbage water aroma) [17].
The 4-ethylphenol (odour threshold value in water at $15 \%$ ethanol, $1000 \mathrm{ppb}$ ) appeared to a high degree in the sharp cider; this compound is associated with spicy, phenolic and bittersweet odours [12].

In relation to the medium-chain fatty acid levels (caprylic and capric), which have a key role in causing stuck fermentations, this was more important in the sharp cider than in the fruity one. However, a greater concentration of long-chain fatty acids (lauric and myristic) was detected in the fruity cider.

\section{Conclusions}

Using the GC-MS method proposed, good recoveries with adequate accuracy for alcohols, esters, lactones, phenols and fatty acids are obtained. However, the short-chain fatty acids are not extracted. The analytical technique outlined is simple, is not time-consuming and the analysis of minor volatile compounds in cider by the proposed method, enables the sensorial profile to be explained.

\section{Acknowledgements}

This work was supported by the Comision Interministerial de Ciencia y Tecnología (Project, ALI 921027-C03) and the Principado of Asturias. 


\section{References}

[1] J. S. Paik, J. Agric. Food Chem. 40, 1822 (1992).

[2] M. Ondarza, A. Sánchez, Chromatographia 30, 16 (1990).

[3] A. A. Williams, H. V. May, O. G. Tucknott, J. Sci. Food Agric. 29, 1041 (1978).

[4] A. A. Williams, H. V. May, O. G. Tucknott, J. Institute Brewing 84, 97 (1978).

[5] J. Breiter, Kontakte 2, 21 (1981).

[6] C. Hubert, P. Brunerie, J. M. Le Quere, J. F. Drilleau, Sciences des Aliments 10, 603 (1990).

[7] V. Gerbi, G. Zeppa, A. Carnacini, Ital. J. Food Sci. 4, 259 (1992).

[8] H. Stashenko, C. Macku, T. Shibamato, J. Agric. Food Chem. 40, 2257 (1992).

[9] S. Wyllie, D. N. Leach, J. Agric. Food Chem. 40, 253 (1992).

[10] $M$. González, Ph. D., University of Madrid, Spain (1986).

[11] C. Edwards, R. Beelman, J. Agric. Food Chem. 38, 216 (1990).

[12] A. Williams, J. Sci. Food Agric. 26, 567 (1975).
[13] H. Nursten, Volatile compounds: The aroma of fruits, in "The Biochemistry of Fruits and their Products", A. C. Hulme, Ed., Academic Press, London, 1970, p. 246.

[14] L. Nykänen, H. Suomalainen, Esters of aliphatic hydroxy and oxo acids, in "Aroma of Beer, Wine and Distilled Alcoholic Beverages", D. Reidel Publishing Company, London, 1983, p. 199.

[15] L. Nykänen, H. Suomalainen, Esters of aliphatic hydroxy and oxo acids, in "Aroma of Beer, Wine and Distilled Alcoholic Beverages", D. Reidel Publishing Company, London, 1983, p. 147.

[16] J. Mangas, M. González, D. Blanco, Z. Lebensm. Unters. Forsch. 197, 522 (1993).

[17] V. Ferreira, Ph. D. University of Zaragoza, Spain (1992).
Received: Jun 30, 1995

Revised manuscript received: Oct 24, 1995 Accepted: Nov 8, 1995 